\title{
Soft Control for Swarm Aggregations
}

\author{
Ying Wang ${ }^{1 *}$, Xuezhi Mao ${ }^{1}$, Liwei Bai ${ }^{2}$ \\ ${ }^{1}$ College of Mathematics and Information Technology, Hebei Normal University of Science and Technology, \\ Qinhuangdao, 066004, China \\ ${ }^{2}$ College of Foreign Languages, Hebei Normal University of Science and Technology \\ Qinhuangdao, 066004, China \\ *Corresponding authors Email: zhuti@ 163.com
}

\begin{abstract}
This paper discusses about the issue of "soft control" for swarms system in Euclidean space, which coordinates the collective behavior of the group by adding a few controlled intelligent agents under the condition of keeping the local rules of the existing agents in the system. It shows that the swarm center will be effectively transferred into an expectant position by putting a few controlled intelligent agents and controlling their initial position or controlling their position for a short time, and the swarm members will converge to a bounded region around the expectant position in a finite time. This paper gives a controlled law for controlled intelligent agents. Simulation testing shows the feasibility of soft control for swarm system.
\end{abstract}

Keywords: Soft control; Swarm; Controlled intelligent agent.

\section{Introduction}

For a long time, it has been observed that certain living beings tend to perform swarming behavior. Examples of swarms include flocks of birds, schools of fish, herds of animals, and colonies of bacteria. This collective behavior has certain advantages, such as threatening predators and increasing the chance of finding food. Recently, many national and international scholars have conducted wide and in-depth researches into various behaviors of swarms, and have achieved many satisfactory results [1-4]. Collective behavior is one of the fundamental and difficult topics of the study of complex systems. We classify the researches on collective behavior into three categories [5].

Given the local rules of the agents, what is the collective behavior of the overall system?

Given the desired collective behavior, what are the local rules for agents?

Given the local rules of agents, how do we control the collective behavior?

The third question is proposed and named "soft control" by Han Jing who works at Institute of Systems Science, Chinese Academy of Sciences, and it coordinates the collective behavior of the group without changing the local rule of the existed agents in the system by increasing one or a few intelligent agents. In some real applications, it is very difficult or even impossible to change the local of agents, such as the behavioral rules of people in panic and the flying strategies of birds. Yet we need to control the system to avoid danger or improve efficiency. Then soft control may be the feasible way to intervene in the collective behavior. Han Jing et al proposed the tactics of "soft control" in Boid model first [5], then Pan Fu-chen et al proposed the tactics of "soft control" in swam system [6], which controlled the swarm center to an expect position by controlling the original position of controlled intelligent agents, given a control law and dis- 
cussed the optimization of convergence time by particle swarm optimization algorithm for several intelligent agents.

This paper puts $H$ controlled intelligent agents into a swarm system with attraction and repulsion functions, leads the swarm to the desired aim by controlling the original position of controlled intelligent agents, gives the different rules of the controlled intelligent agents, and analyses of stability and proof. Furthermore, this paper considers a more general attraction/repulsion function, and introduces a new control method, which can choose the initial position of controlled intelligent agents optionally, and then lead the center of the swarm to the desired aim by regulating the position of controlled intelligent agents gradually. Since swarm system is a multi-agent system, adding one or a few agents will not affect it. Simulation testing further shows the feasibility of soft control for swarms system.

\section{Model of Swarm Systems}

We consider a swarm of $M$ individuals (members) in an $\mathrm{N}$-dimensional Euclidean space and model the individuals as points and ignore their dimensions. The position of member $i$ of the swarm is described by $\chi^{i} \subset R^{n}$. We assume synchronous motion and no time delays, i.e., all the members move simultaneously and know the exact position of all the other members. The equation of motion of individual $i$ is given by [1]

$$
\dot{x}^{i}=\sum_{j=1, j \neq i}^{M} g\left(x^{i}-x^{j}\right), i=1,2, \ldots, M
$$

where $g($.$) represents the function of attraction and$ repulsion between the members. In other words, the direction and magnitude of motion of each member is determined as a weighted sum of the attraction and repulsion of all the other members. The attraction/ repulsion function that we consider is

$$
g(y)=-y\left(a-b \exp -\frac{\|y\|^{2}}{c}\right)
$$

Where $a, b$, and $c$ are positive constants such that $b>a$, and $\|y\|$ is the Euclidean norm given by $\|y\|=$ $\sqrt{y^{T} y}$. The parameter $a$ represents the attraction and the term $b \exp (-\|y\| / c)$ represents the repulsion.

By equating $-y\left(a-b \exp -\frac{\|y\|^{2}}{c}\right)=0$ it can be easily found that $\mathrm{g}(\mathrm{y})$ switches sign at the set of points defined as $Y=\left(y=0 o r\|y\|=\delta=\sqrt{c \ln \frac{b}{a}}\right)$ The distance is the distance where the attraction and repulsion balance.
Definition 1 [1] The center of the swarm members is

$$
\bar{x}=\frac{1}{M} \sum_{i=1}^{M} x^{i}
$$

$$
\begin{aligned}
& \text { Let } g_{1}\left(x^{i}-x^{j}\right)=a-b \exp \left(-\frac{\left\|x^{i}-x^{j}\right\|^{2}}{c}\right) \text { then } \\
& \qquad \overline{\dot{x}}=-\frac{1}{M} \sum_{i=1}^{M} \sum_{j=1, j \neq i}^{M}\left(x^{i}-x^{j}\right) g_{1}\left(x^{i}-x^{j}\right)=0
\end{aligned}
$$

Hence the swarm center $\bar{x}$ is stationary for all $t$.

\section{Soft Control for Swarm Systems}

Considering that we add $\mathrm{H}$ controlled intelligent agents in model (1), whose position is optionally controllable, and the guise of controlled intelligent agents is the same as ordinary agents in the swarm, the existing agents thus still treat the controlled intelligent agent as an ordinary agent.

The position of controlled intelligent agent $\mathrm{s}$ is described by $x^{M+s} \in R^{n}(s=1,2, \ldots, H)$, and $x_{0}^{M+s} \in R^{n}(s=$ $1,2, \ldots, H)$ represents the initial position of controlled intelligent agents.

To control the swarm system and make the center of the swarm members to converge at the expectant position, we adopt the following rule to controlled intelligent agents

$$
\begin{array}{r}
x_{0}^{M+s}=\frac{2 s}{H(H+1)}\left[(M+H) x^{e}-M \bar{x}_{M}\right], \\
s=1,2, \ldots, H
\end{array}
$$

where $x^{e}$ is the expectant center, $\bar{x}_{M}=\frac{1}{M} \sum_{i=1}^{M} x^{i}$ is the initial center of the swarm system (barring controlled intelligent agents).

Hence, swarm model (1) is considered as

$$
\dot{x}^{i}=\sum_{j=1, j \neq i}^{M+H} g\left(x^{i}-x^{j}\right), i=1,2, \ldots, M+H
$$

Note: The rules to controlled intelligent agents are not the only one. Some other rules please see [6].

Theorem 1 For any original agents of swarm system, adopt the rule (3) to controlled intelligent agents. The center of system will be transferred to the expectant position $x^{e}$, and the swarm center is stationary for all $t$.

Proof Considerring the model (4) which is added $H$ controlled intelligent agent, the center of $\mathrm{M}+\mathrm{H}$ swarm 
members is

$$
\begin{aligned}
& \bar{x}_{M+H}=\frac{1}{M+H}\left(\sum_{i=1}^{M} x^{i}+\sum_{s=1}^{H} x_{0}^{M+s}\right) \\
& =\frac{1}{M+H}\left\{\sum_{i=1}^{M} x^{i}+\right. \\
& \left.+\sum_{s=1}^{H} \frac{2 s}{H(H+1)}\left[(M+H) x^{e}-M \bar{x}_{M}\right]\right\} \\
& =\frac{1}{M+H}\left\{\sum_{i=1}^{M} x^{i}+\right. \\
& \left.+\frac{2}{H(H+1)}\left[(M+H) x^{e}-\sum_{i=1}^{M} x^{i}\right] \sum_{s=1}^{H} s\right\} \\
& =\frac{1}{M+H}\left\{\sum_{i=1}^{M} x^{i}+\right. \\
& \left.+\frac{2}{H(H+1)}\left[(M+H) x^{e}-\sum_{i=1}^{M} x^{i}\right] \frac{H(H+1)}{2}\right\} \\
& =x^{e}
\end{aligned}
$$

which proves the theorem.

Theorem 1 shows that center of swarm can be transferred effectively by adding $H$ controlled intelligent agents and adopting the soft control rule (3). It is to say that we can have the group collect any expectant position.

Definition 2 [1] A swarm member $i$ is called a free agent at time $t$ if

$$
\left\|x(t)-x^{j}(t)\right\|>\delta, \forall j \in S, j \neq i
$$

where $S=1,2, \ldots, M+H$ is the set of members of the swarm.

Theorem 2 Let $e^{i}=x^{i}-x^{e}$, and assume that a member $i$ of the swarm described by the model in (4) is a free agent at time $t$ and that its distance to the center $x^{e}$ of the swarm is greater than $\delta$, i.e., $\left\|e^{i}\right\|=\left\|x^{i}-x^{e}\right\|>$ $\delta$, then, at time $t$, its motion is toward the center $x^{e}$.

Proof From the definition of the center of the swarm, we have

$$
\sum_{j=1}^{M+H} x^{j}=(M+H) \bar{x}_{M+H}=(M+H) x^{e}
$$

Subtracting from both sides $(M+H) x^{i}$, we obtain

$$
\sum_{j=1}^{M+H}\left(x^{i}-x^{j}\right)=(M+H)\left(x^{i}-x^{e}\right)=(M+H) e^{i}
$$

Then, the motion of member $i$ can be represented as

$$
\begin{aligned}
\dot{x}^{i}= & -\sum_{j=1, j \neq i}^{M+H}\left(x^{i}-x^{j}\right)\left[a-b \exp \left(-\frac{\left\|x^{i}-x^{j}\right\|^{2}}{c}\right)\right] \\
= & -a(M+H) e^{i}+ \\
& +b \sum_{j=1, j \neq i}^{M+H} \exp \left(-\frac{\left\|x^{i}-x^{j}\right\|^{2}}{c}\right)\left(x^{i}-x^{j}\right)
\end{aligned}
$$

Choosing the Lyapunov function candidate for member $i$ as $V_{i}=\frac{1}{2} e^{i T} e^{i}$, then

$$
\begin{aligned}
\dot{V}_{i}= & e^{i T} \dot{e}^{i}=e^{i T}\left(\dot{x}^{i}-\dot{x}^{e}\right)=e^{i T} \dot{x}^{i} \\
= & e^{i T}\left[-a(M+H) e^{i}+\right. \\
& \left.+b \sum_{j=1, j \neq i}^{M+H} \exp \left(-\frac{\left\|x^{i}-x^{j}\right\|^{2}}{c}\right)\left(x^{i}-x^{j}\right)\right] \\
& \leq-a(M+H)\left\|e^{i}\right\|^{2}+ \\
& +b \sum_{j=1, j \neq i}^{M+H} \exp \left(-\frac{\left\|x^{i}-x^{j}\right\|^{2}}{c}\right)\left\|x^{i}-x^{j}\right\|\left\|e^{i}\right\|
\end{aligned}
$$

Since $\left\|x^{i}-x^{j}\right\|>\delta, \forall \in S, j \neq i$, it should be noted that for that range the function $\exp \left(-\frac{\left\|x^{i}-x^{j}\right\|^{2}}{c}\right) \| x^{i}-$ $x^{j} \|$ is a decreasing function of the distance with the maximum $\delta \exp \left(-\frac{\delta^{2}}{c}\right)$ occurring at $\left\|x^{i}-x^{j}\right\|=\delta$. With these facts, we have

$$
\begin{aligned}
\dot{V}_{i} & \leq-a\left\|e^{i}\right\|^{2}- \\
& -(M+H-1)\left[a\left\|e^{i}\right\|-b \delta \exp \left(-\frac{\delta^{2}}{c}\right)\right]\left\|e^{i}\right\|
\end{aligned}
$$

Because $\left\|e^{i}\right\| \geq \delta$ and $\frac{b}{a} \exp \left(-\frac{\delta^{2}}{c}\right)=1$, the second term is not positive, therefore, we have

$$
\dot{V}_{i} \leq-a\left\|e^{i}\right\|^{2}=-a V_{i}
$$

which proves the theorem.

Theorem 3 Considering the swarm described by the model in (4) as time progresses, all the members of the swarm will converge to a hyperball

$$
B_{\varepsilon}\left(x^{e}\right)=x:\left\|x-x^{e}\right\| \leq \varepsilon
$$

where

$$
\varepsilon=\frac{b}{a} \sqrt{\frac{c}{2}} \exp \left(-\frac{1}{2}\right)
$$

Moreover, the convergence will occur in finite time bounded by

$$
\bar{t}=\max _{i \in S}-\frac{1}{2 a} \ln \left(\frac{\varepsilon^{2}}{2 V_{i}(0)}\right)
$$


Proof Choose any swarm member $i$. Let $V_{i}=\frac{1}{2} e^{i T} e^{i}$ be the corresponding Lyapunov function. From theorem 2, we have

$$
\begin{aligned}
\dot{V}_{i} & \leq-a(M+H)\left\|e^{i}\right\|^{2}+ \\
& b \sum_{j=1, j \neq i}^{M+H} \exp \left(-\frac{\left\|x^{i}-x^{j}\right\|^{2}}{c}\right)\left\|x^{i}-x^{j}\right\|\left\|e^{i}\right\|
\end{aligned}
$$

Note that each of the functions $\exp \left(-\frac{\left\|x^{i}-x^{j}\right\|^{2}}{c}\right) \| x^{i}-$ $x^{j} \|$ is a bounded function,

$$
\left.\exp -\frac{\left\|x^{i}-x^{j}\right\|^{2}}{c}\right)\left\|x^{i}-x^{j}\right\| \leq \sqrt{\frac{c}{2}} \exp \left(-\frac{1}{2}\right)
$$

whose maximum occurs at $\left\|x^{i}-x^{j}\right\|=\sqrt{\frac{c}{2}}$. Substituting this in the equation (6), we obtain

$$
\begin{aligned}
\dot{V}_{i} \leq & -a(M+H)\left\|e^{i}\right\|^{2}+ \\
& +b \sum_{j=1, j \neq i}^{M+H} \exp \left(-\frac{1}{2}\right) \sqrt{\frac{c}{2}}\left\|e^{i}\right\| \\
= & -a(M+H)\left\|e^{i}\right\|^{2}+ \\
& +b(M+H-1) \exp \left(-\frac{1}{2}\right) \sqrt{\frac{c}{2}}\left\|e^{i}\right\|
\end{aligned}
$$
0 .

If $\left\|e^{i}\right\|>\frac{b(M+H-1)}{a(M+H)} \sqrt{\frac{c}{2}} \exp \left(-\frac{1}{2}\right)$ then we have $\dot{V}_{i}<$

Define $\varepsilon=\frac{b}{a} \sqrt{\frac{c}{2}} \exp \left(-\frac{1}{2}\right)$ and note that

$$
\varepsilon>\frac{b(M+H-1)}{a(M+H)} \sqrt{\frac{c}{2}} \exp \left(-\frac{1}{2}\right)
$$

This implies that as $t \rightarrow \infty, e^{t}$ converges within the ball around $x^{e}$ defined by $\frac{b(M+H-1)}{a(M+H)} \sqrt{\frac{c}{2}} \exp \left(-\frac{1}{2}\right)$.

Since $\varepsilon>\frac{b(M+H-1)}{a(M+H)} \sqrt{\frac{c}{2}} \exp \left(-\frac{1}{2}\right)$ we have $e^{i} \rightarrow B_{\varepsilon}$.

Since member $i$ was an arbitrary one, the result holds for all the members. To prove the finite-time convergence, note that $\left\|e^{i}\right\| \geq \varepsilon$, we have

$$
\dot{V}_{i} \leq-a\left\|e^{i}\right\|^{2}=-2 a V_{i}
$$

Therefore, the solution of $V_{i}$ satisfies

$$
V_{i}(t) \leq V_{i}(0) e^{-2 a t}
$$

which can be shown by crossing the $\left\|e^{i}\right\|=\varepsilon$ boundary in a time bounded by

$$
t_{i} \leq-\frac{1}{2 a} \ln \left(\frac{\varepsilon^{2}}{2 V_{i}(0)}\right)
$$

and this proves the theorem.
Theorem 3 shows only the region where the swarm members will converge and provide a bound on the size of the swarm. It does not, however, say anything about whether the swarm members will stop their motion or start an oscillatory motion within the regionsothis issue needs to be investigated further. We first define the state $\mathrm{x}$ of the system as the vector of the positions of the swarm members $x=\left[x^{1 T}, x^{2 T}, \ldots, x^{(M+H) T}\right]^{T}$. Let the invariant set of equilibrium points be

$$
\Omega_{e}=\{x: \dot{x}=0\}
$$

We will prove that as $t \rightarrow \infty$ the state $x(t)$ converges to $\Omega_{e}$, i.e., the configuration of the swarm members converges to a constant arrangement.

Definition 3 [4] The swarm system (4) is completely stable if every solution to it converges to an equilibrium point of the system.

Theorem 4 The swarm system (4) is completely stable.

Proof We choose the Lyapunov function

$$
\begin{aligned}
& J(x)=\frac{1}{2} \sum_{i=1}^{M+H-1} \sum_{j=i+1}^{M+H} {\left[a\left\|x^{i}-x^{j}\right\|^{2}+\right.} \\
&\left.+b c \exp \left(-\frac{\left\|x^{i}-x^{j}\right\|^{2}}{c}\right)\right]
\end{aligned}
$$

which is an artificial potential function. Then, one can show that the gradient of $J(x)$ with respect to each $x^{i}$ is given by $\nabla_{x^{i}} J(x)=-\dot{x}^{i}$. Now, we take the time derivative of the Lyapunov function along the motion of the system and obtain

$$
\begin{aligned}
\dot{J}(x) & =\left[\nabla_{x} J(x)\right]^{T} \dot{x}=\sum_{i=1}^{M+H}\left[\nabla_{x^{i}} J(x)\right]^{T} \dot{x}^{i} \\
& =\sum_{i=1}^{M+H}\left[-\dot{x}^{i}\right] \dot{x}^{i}=-\sum_{i=1}^{M+H}\left\|\dot{x}^{i}\right\|^{2} \leq 0
\end{aligned}
$$

for all $t$. Then, using the LaSalle's Invariance Principle we conclude that as $t \rightarrow \infty$ the state $x$ converges to the largest invariant subset of the set defined as

$$
\Omega=\{x: \dot{J}(x)=0\}=\{x: \dot{x}=0\}=\Omega
$$

Since each point in $\Omega_{e}$ is an equilibrium, $\Omega_{e}$ is an invariant set and this proves the result.

\section{Further Explorations}

In Sections 2 and 3, we considered a specific attraction/repulsion function $g(y)$ as defined in (2), put $H$ controlled intelligent agents into the swarm system 
and led the center of swarm to the desired aim by controlling the initial position of controlled intelligent agents. However, due to the complexity of the interactions among agents, sometimes, we can not obtain its explicit expression; furthermore, it is very difficult to control the initial position of controlled intelligent agents when we put it into the swarm system.

In this section, we will consider a more general attraction/repulsion function $g(y)$, and introduce a new control method, which can choose the initial position of controlled intelligent agents optionally, and then lead the center of the swarm to the desired aim by regulating the position of controlled intelligent agents gradually.

Following [3,4], we have the assumptions listed below:

Assumption 1 The attraction/repulsion function $g(y)$ is the form

$$
g(y)=-y\left[g_{a}(\|y\|)-g_{r}(\|y\|)\right], y \in R^{n}
$$

where $g_{a}: R^{+} \rightarrow R^{+}$represents (the magnitude of) the attraction term, whereas $g_{r}: R^{+} \rightarrow R^{+}$represents (the magnitude of) the repulsion term, and $\|y\|$ is the Euclidean norm given by $\|y\|=\sqrt{y^{T} y}$.

Assumption 2 There are some finite positive constants $a, b$, for any $y \in R^{n}$,

$$
g_{a}(\|y\|) \geq a, \operatorname{and}_{r}(\|y\|) \leq \frac{b}{\|y\|}
$$

That is, we assume a linearly bounded from below attraction and a bounded repulsion.

Assumption 3 There exist corresponding functions $J_{a}: R^{+} \rightarrow R^{+}$and $J_{r}: R^{+} \rightarrow R^{+}$for any $y \in R^{n}$,

$$
\begin{aligned}
& \nabla_{y} J_{a}(\|y\|)-y g_{a}(\|y\|) \\
& \nabla_{y} J_{r}(\|y\|)-y g_{r}(\|y\|)
\end{aligned}
$$

In other words, we assume that the attraction and repulsion among the swarm members are governed by potential fields $J_{a}(\|y\|)$ and $J_{r}(\|y\|)$. In this case, the motion of the individuals moves toward each other along the gradient of these fields.

Under Assumptions 1, 2 and 3, for controlling the center of the swarm members to the expectant position $x^{e}$, we consider adding $H$ controlled intelligent agents in model (1), the position of controlled intelligent agent $s$ is described by $x^{M+s} \in R^{n}$, and its initial position $x^{M+s} \in R^{n}(s=1,2, \ldots, H)$ is optional. Hence, swarm model (1) is considered as model (4), but the center of members may not be $x^{e}$. To control the center $x^{e}$ and avoid quick change of the position of controlled intelligent agents, we adopt the following rules to controlled intelligent agents from time 0 to time $t_{0}$

$$
\begin{gathered}
x^{M+S}=[1-\lambda(t)] x^{M+s}+\frac{2 s \lambda(t)}{H(H+1)}\left[(M+H) x^{e}-\right. \\
\left.-M \bar{x}_{M}\right] \quad s=1,2, \ldots, H
\end{gathered}
$$

where $\lambda(t)$ is bounded, $\lambda(t)$ changes continuously to 1 from 0 when time changes to $t_{0}$ from 0 , and the control rule is canceled when $t>t_{0}$. Then the controlled swarm system is expressed as If $t \leq t_{0}$, then

$$
\left\{\begin{array}{c}
\dot{c}^{i}=\sum_{j=1, j \neq i}^{M} g\left(x^{i}-x^{j}\right), \quad i=1,2, \ldots, M \\
x^{M+S}=[1-\lambda(t)] x^{M+s}+\frac{2 s \lambda(t)}{H(H+1)}\left[(M+H) x^{e}-\right. \\
\left.-M \bar{x}_{M}\right], \quad s=1,2, \ldots, H
\end{array}\right.
$$

and $t>t_{0}$, then

$$
\dot{x}^{i}=\sum_{j=1, j \neq i}^{M+H} g\left(x^{i}-x^{j}\right), \quad i=1,2, \ldots, M+H
$$

Theorem 5 For any original agents of swarm system (11), adopt the rule (10) to controlled intelligent agents, the center of system will be transferred to the expectant position $x^{e}$ when $t>t_{0}$, and the swarm center is stationary for all $\mathrm{t}\left(t>t_{0}\right)$.

Proof Because $\lambda(t)=1$ when $t=t_{0}$, and the rule (10) becomes

$$
x^{M+s}=\frac{2 s}{H(H+1)}\left[(M+H) x^{e}-M \bar{x}_{M}\right]
$$

$(s=1,2, \ldots, H)$ at this time. Having the method as Theorem 1, we will prove that the center of members gets to $x^{e}$. When $t>t_{0}$ the model (11) becomes

$$
\dot{x}^{i}=\sum_{j=1, j \neq i}^{M+H} g\left(x^{i}-x^{j}\right), \quad i=1,2, \ldots, M+H
$$

Under Assumption 1, the swarm center is stationary for all $t$ when $t>t_{0}$, because the time derivative of center is given by

$$
\begin{gathered}
\dot{\bar{x}}=-\frac{1}{M+H} \sum_{i=1}^{M+H} \sum_{j=1, j \neq i}^{M+H}\left[g_{a}\left(\left\|x^{i}-x^{j}\right\|\right)-\right. \\
\left.-g_{r}\left(\left\|x^{i}-x^{j}\right\|\right)\right]\left(x^{i}-x^{j}\right) \\
=-\frac{1}{M+H} \sum_{i=1}^{M+H} \sum_{j=1, j \neq i}^{M+H}\left[\left[g_{a}\left(\left\|x^{i}-x^{j}\right\|\right)-\right.\right. \\
\left.-g_{r}\left(\left\|x^{i}-x^{j}\right\|\right)\right]\left(x^{i}-x^{j}\right)+\left[g_{a}\left(\left\|x^{i}-x^{j}\right\|\right)-\right. \\
\left.\left.\quad-g_{r}\left(\left\|x^{i}-x^{j}\right\|\right)\right]\left(x^{j}-x^{i}\right)\right]=0
\end{gathered}
$$

Theorem 6 Considering the swarm described by the model in (11), as time progresses from $t_{0}$, all the members of the swarm will enter and stay in the bounded region

$$
\Omega\left(x^{e}\right)=x:\left\|x-x^{e}\right\| \leq \varepsilon
$$


where $\varepsilon=\frac{b}{a}$. Moreover, the convergence will occur in finite time bounded by

$$
\bar{t}=\max _{i \in S}\left(-\frac{1}{2 a} \ln \left(\frac{\varepsilon^{2}}{2 V_{i}(0)}\right)\right)
$$

where $S$ is the set of all individuals $S=1, \ldots, M+H$.

Proof Let $e^{i}=x^{i}-x^{e}$, we have

$$
\dot{e}^{i}=\dot{x}^{i}-\dot{x}^{e}=\dot{x}^{i}
$$

Choosing the Lyapunov function candidate for member $i$ as $V_{i}=\frac{1}{2}\left\|x^{i}\right\|^{2}=\frac{1}{2} e^{i T} e^{i}$. Then note that

$$
\begin{aligned}
\sum_{j=1 j \neq i}^{M+H}\left(x^{i}-x^{j}\right) & =\sum_{j=1}^{M+H}\left(x^{i}-x^{j}\right) \\
& =(M+H) x^{i}-\sum_{j=1}^{M+H} x^{j} \\
& =(M+H) x^{i}-(M+H) \bar{x} \\
& =(M+H) x^{i}-(M+H) x^{e} \\
& =(M+H) e^{i}
\end{aligned}
$$

we have

$$
\begin{aligned}
\dot{V}_{i}= & e^{i T} e^{i} \\
= & -\sum_{j=1, j \neq i}^{M+H}\left[g_{a}\left(\left\|x^{i}-x^{j}\right\|\right)-g_{r}\left(\left\|x^{i}-x^{j}\right\|\right)\right]\left(x^{i}-x^{j}\right) e^{i} \\
\leq & -a \sum_{j=1, j \neq i}^{M+H}\left(x^{i}-x^{j}\right)^{T} e^{i}+ \\
& \sum_{j=1, j \neq i}^{M+H} g_{r}\left(\left\|x^{i}-x^{j}\right\|\right)\left(x^{i}-x^{j}\right) e^{i} \\
= & -a(M+H)\left\|e^{i}\right\|^{2}+ \\
& \sum_{j=1, j \neq i}^{M+H} g_{r}\left(\left\|x^{i}-x^{j}\right\|\right)\left(x^{i}-x^{j}\right) e^{i} \\
\leq & -a(M+H)\left\|e^{i}\right\|^{2}\left[\left\|e^{i}\right\|-\right. \\
& \left.\frac{1}{a(M+H)} \sum_{j=1, j \neq i}^{M+H} g_{r}\left(\left\|x^{i}-x^{j}\right\|\right)\left\|x^{i}-x^{j}\right\|\right] \\
\leq & -a(M+H)\left\|e^{i}\right\|^{2}\left[\left\|e^{i}\right\|-\frac{1}{a(M+H)} \sum_{j=1, j \neq i}^{M+H} b\right] \\
= & -a(M+H)\left\|e^{i}\right\|^{2}\left[\left\|e^{i}\right\|-\frac{(M+H-1) b}{a(M+H)}\right]
\end{aligned}
$$

which implies that $\dot{V}_{i}<0$ as long as $\left\|e^{i}\right\|>\frac{(M+H-1) b}{a(M+H)}$. This, on the other hand, implies that as $t \rightarrow \infty$ asymptotically we have

$$
\left\|e^{i}\right\| \leq \frac{(M+H-1) b}{a(M+H)}<\frac{b}{a} \varepsilon
$$

which provides a bound on the maximum ultimate swarm size. Moreover, it should be noted that for $\left\|e^{i}\right\| \geq \varepsilon$ we have $\dot{V}_{i} \leq-a\left\|e^{i}\right\|^{2}=-2 a V_{i}$; it can show that we will have $\left\|e^{i}\right\|<\varepsilon$ for all $i$ in a finite time bounded by

$$
\bar{t}=\max _{i \in S}\left(-\frac{1}{2 a} \ln \left(\frac{\varepsilon^{2}}{2 V_{i}(0)}\right)\right)
$$

where $S$ is the set of all individuals $S=1, \ldots, M+H$.

Theorem 7 The swarm system (11) is completely stable.

Proof Under Assumption 3, the motion of each individual is given by

$$
\dot{x}^{i}=-\sum_{j=1, j \neq i}^{M+H}\left[\nabla_{x^{i}} J_{a}\left(\left\|x^{i}-x^{j}\right\|\right)-\nabla_{x^{i}} J_{r}\left(\left\|x^{i}-x^{j}\right\|\right)\right]
$$

for all $i=1,2, M+H$.

Now we choose the Lyapunov function as

$J(x)=\sum_{j=1, j \neq i}^{M+H} \sum_{j=i+1}^{M+H}\left[\nabla_{x^{i}} J_{a}\left(\left\|x^{i}-x^{j}\right\|\right)-\nabla_{x^{i}} J_{r}\left(\left\|x^{i}-x^{j}\right\|\right)\right]$

Taking the gradient of $J(x)$ with respect to the position $x^{i}$ of individual $i$, we obtain

$$
\begin{aligned}
\nabla_{x^{i}} J(x) & =\sum_{j=1, j \neq i}^{M+H}\left[\nabla_{x^{i}} J_{a}\left(\left\|x^{i}-x^{j}\right\|\right)-\nabla_{x^{i}} J_{r}\left(\left\|x^{i}-x^{j}\right\|\right)\right] \\
& =-\dot{x}^{i}
\end{aligned}
$$

Now, taking the time derivative of the Lyapunov function along with the motion of the system we obtain

$$
\begin{aligned}
\dot{J}(x) & =\left[\nabla_{x} J(x)\right]^{T} \dot{x}=\sum_{i=1}^{M+H}\left[\nabla_{x^{i}} J(x)\right]^{T} \dot{x}^{i} \\
& =\sum_{i=1}^{M+H}\left[-\dot{x}^{j}\right]^{T} \dot{x}^{j}=-\sum i=1^{M+H}\left\|\dot{x}^{i}\right\|^{2}<0
\end{aligned}
$$

for all $t>t_{0}$. Hence, $J(x)$ decreases in $t$ unless $\dot{x}^{i}=0$ for all $i$. To conclude the complete stability of the system, we recall that, by Theorem 6 , every solution $x(t)$ of system (11) will converge to the compact set $\Omega\left(x^{e}\right)$. Thus all solutions of the system are bounded. Therefore, by the LaSalle's Invariance Principle we conclude that as $t \rightarrow \infty$ the solution $x(t)$ converges to the largest positively invariant subset of the set defined by $E=\{x: \dot{J}(x)=0\}$.

It is clear from the discussion above that $E=\Omega_{e}$ and is positively invariant with respect to the system. Thus, $x(t) \rightarrow \Omega_{e}$ as $t \rightarrow \infty$. 


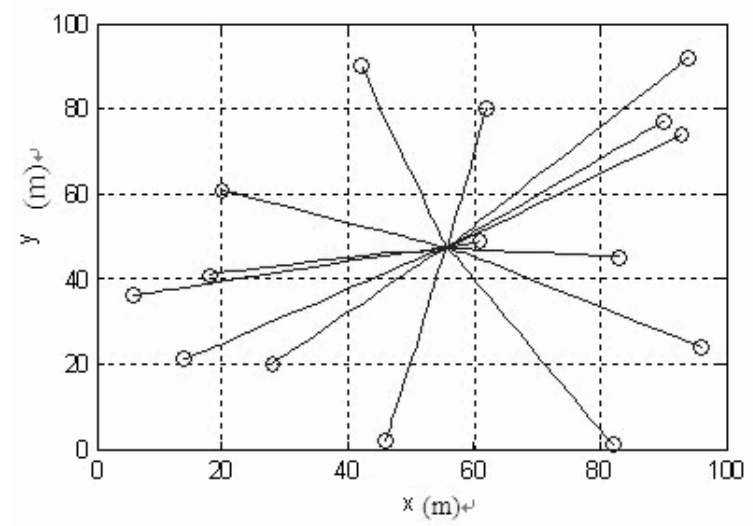

Figure 1. The trajectories of fifteen agents without controlled intelligent agent

\section{Simulation}

In this section, we present some numerical simulations for the soft control for swarm systems in order to illustrate the theoretic results obtained in the previous sections.

In these simulations, the attraction/repulsion function is taken in the form of (2) with parameters $a=$ $3, b=8$, and $c=0.2$. Choose 2-dimensional space as the practical space, let $M=15, H=3$. The original positions of swarm members are obtained randomly by computer.

Fig. 1 shows the trajectories of the swarm members without any controlled intelligent agent. Fig. 2 shows the trajectories of the members with three controlled intelligent agents in the swarm. Adopt the rule (3) to controlled intelligent agents, the intention is transferring the center of system to the expectant position $(40,60)$. The red broken lines describe the motion of controlled intelligent agent, and the sign "o" represents the original position of agents.

Fig. 3 shows the trajectories of the members with three controlled intelligent agents in the swarm. The initial positions of controlled intelligent agents are optional. Then adopt the rule (10) to controlled intelligent agents to transfer the center of system to the expectant position $(40,60)$. Let $\lambda(t)=10 t$ and $t_{0}=0.1$. Fig. 4 shows the trajectory of the center, it may be different if one chooses different $\lambda(t)$.

The result of simulations shows: add 3 controlled intelligent agents in swarm model, and adopt the rule (3) or (10) to controlled intelligent agents, the center of swarm members will be effectively transferred to the expectant position $(40,60)$, and the swarm systems tend to stability gradually after brief instability.

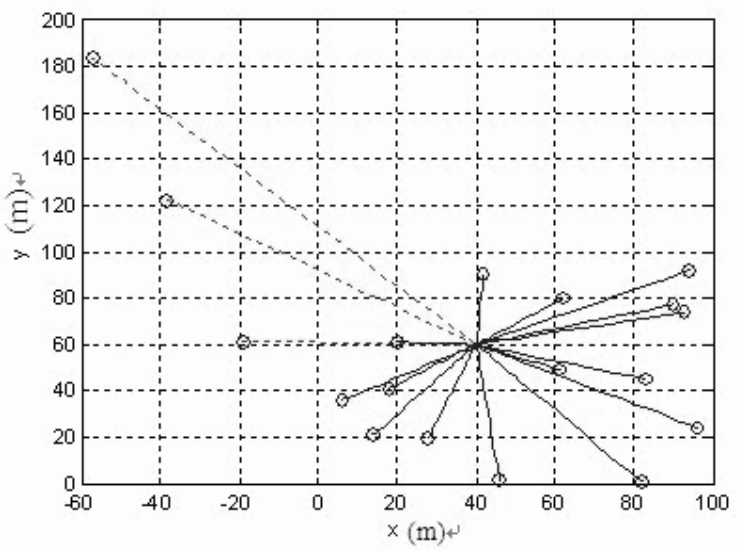

Figure 2. The trajectories of the swarm members with three controlled intelligent agents

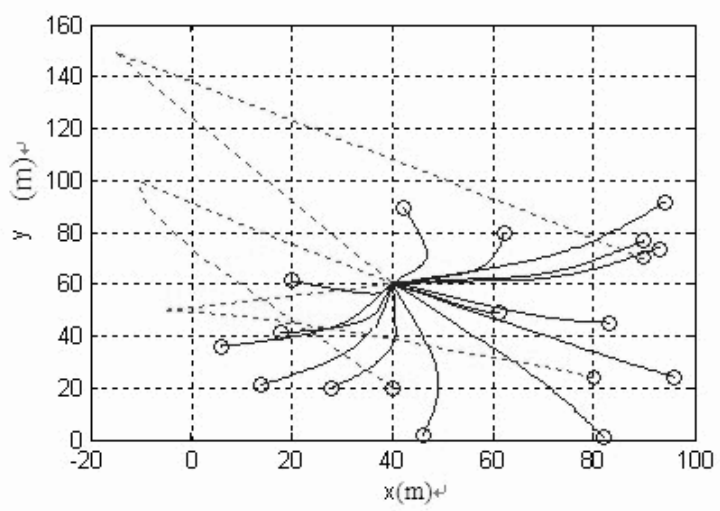

Figure 3. The trajectories of the swarm members with three controlled intelligent agents

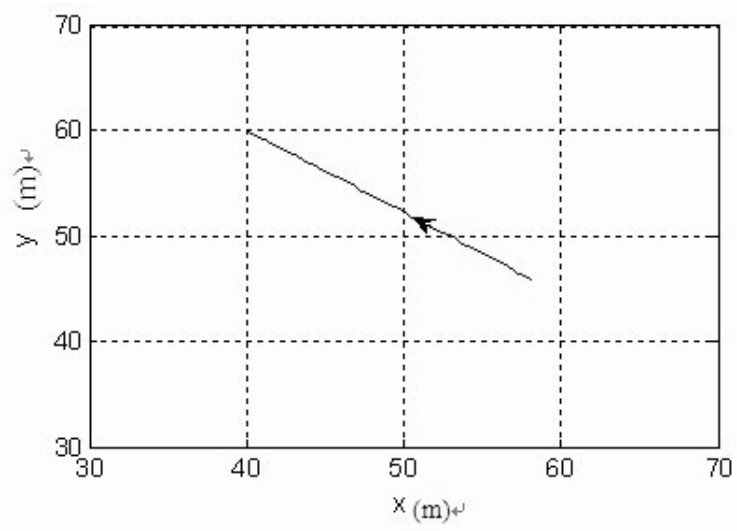

Figure 4. The trajectory of the center 


\section{Conclusion}

This paper focuses on "soft control" for swarms system, i.e., control the center of swarm members by adding $H$ controlled intelligent agents but without changing the local rule of the existing agents in the system. Simulation testing shows the feasibility of soft control for swarms system.

Soft control for swarms system is a new research direction, and there are also lots of problems to be tackled, such as the optimization of number and position of controlled intelligent agents, the expansion of control means, the design of controlled intelligent agent and so on. Soft control, as a new control method, will provide vaster foreground for the research of complex systems.

\section{References}

[1] Gazi V, Passino KM., "Stability analysis of swarms", IEEE Trans on Automatic Control, 2003, 48(4): 692697.

[2] Gazi V, Passino KM, "Stability analysis of swarms in an environment with an attractant /repellent profile", Proceedings of American Control Conference. 2002: 1819-1824.

[3] Gazi V, Passino KM, "A class of attraction/repulsion functions for stable swarm aggregations", International Journal of Control, 2004, 77(18): 1567-1579.

[4] Chu TG, Wang L, and Chen TW, "Self-organized motion in anisotropic swarms", Journal of Control Theory and Applications, 2003, 1: 77-81.

[5] Han J, Li M, and Guo L, "Soft control on collective behavior of a group of automamous agents by a shill agent", Journal of Systems Science and Complexity, 2006,19(1): 54-62.

[6] Pan FC, Chen XB, and Li L, "Soft control of swarms system”, Control and Decision, 200823(8)953-956.

[7] Wang Y, Mao XZ, and Liu JP. "Soft control for swarm systems with simple attraction and repulsion functions [C]". 2009 Second International Conference on Intelligent Networks and Intelligent Systems, 2009: 482-485.

[8] Mao XZ, Xu Y, Wang Y. "Soft control for swarm systems based on attraction and repulsion functions [C]". 2009 Second International Conference on Intelligent Networks and Intelligent Systems, 2009: 47.

[9] Slotine JE, Li W, Cheng DZ (translation). Applied Nonlinear Control, Beijing: China Machine Press, 20064.

[10] Xue DY. Computer Aided Control Systems Design Using MATLAB Language (Second Edition), Beijing: Tsinghua University Press. 20063. 